\title{
Neutral alleles at hybrid sterility loci of Oryza glaberrima from AA genome relatives in Genus Oryza
}

\author{
Jing $\mathrm{Li}^{\dagger 1)}$, Jiawu Zhou ${ }^{\dagger 1)}$, Peng Xu${ }^{1)}$, Xianneng Deng1), Wei Deng ${ }^{1)}$, Mingzhu He ${ }^{2)}$, Ying Yang1), Yu Zhang') \\ and Dayun Tao*1) \\ 1) Food Crops Research Institute, Yunnan Academy of Agricultural Sciences (YAAS), Kunming 650200, P. R. China \\ 2) East China Normal University, Shanghai 200241, P. R. China
}

\begin{abstract}
Hybrid sterility between Oryza sativa and O. glaberrima is a main reproduction barrier when transferring the favorable alleles from $O$. glaberrima to $O$. sativa and it happens due to allelic interaction at sterility loci. Neutral alleles at each locus have the potential to overcome the sterility between the two cultivated rice species. In this study, an $O$. sativa cultivar Dianjingyou 1 (DJY1) and its near-isogenic lines (NILs) harboring the single sterility allele $S 1$-glab, $S 19$-glab, $S 20$-glab, $S 37$-glab, $S 38$-glab and $S 39$-glab as the tested lines were crossed with $O$. glaberrima, O. rufipogon, O. nivara, O. glumaepatula, O. barthii, O. meridionalis and $O$. sativa so as to detect the neutral alleles of these loci. Pollen fertility was investigated in the paired $\mathrm{F}_{1} \mathrm{~s}$ based on two seasons' result and genotypic segregation was also analyzed in some $F_{2}$ populations to confirm the results of pollen fertility investigation. The neutral alleles of $S 38-\mathrm{n}$ and $S 39-\mathrm{n}$ were identified based upon the pollen fertility and genotypic segregation analysis for the first time. The neutral alleles of sterility loci detected from present report have the potential to know of the nature of interspecific hybrid sterility, and to overcome the interspecific hybrid sterility between $O$. sativa and $O$. glaberrima.
\end{abstract}

Key Words: hybrid sterility, interspecific hybrid, allelic variation, neutral allele, Oryza glaberrima, Oryza nivara.

\section{Introduction}

Asian cultivated rice (Oryza sativa L.) is a major food crop around the world. But its breeding was faced with problems of the narrow genetic diversity and the bottleneck of further yield increase (Tanksley and McCouch 1997). African cultivated rice (O. glaberrima Steud.) is deemed to be a potential source of useful genes for Asian cultivated rice improvement as both Asian and African cultivated species have the same AA genome and similar sequence arrangement (Chang 1976, Ohmido and Fukui 1995). However, there are harsh reproductive barriers in the interspecific hybrids between O. glaberrima and O. sativa (Sano 1990). The $F_{1}$ hybrids barely have a fertile pollen grain and as a result the valuable genes contained in $O$. glaberrima naturally disappear along with hybrid sterility. Thus, hybrid sterility is one of the main hindrances against the utilization of

Communicated by Hiroshi Kato

Received January 11, 2018. Accepted March 11, 2018.

First Published Online in J-STAGE on June 29, 2018.

*Corresponding author (e-mail: taody12@aliyun.com)

${ }^{\dagger}$ These authors contributed equally to this work useful genes from the African cultivated rice to Asian cultivated rice.

The phenomenon of $F_{1}$ hybrid sterility is widely observed in intersubspecific and interspecific hybrids. More than 50 loci controlling the hybrid sterility were identified and a few of them were cloned in rice (Chen et al. 2008, Kubo et al. 2015, Long et al. 2008, Mizuta et al. 2010, Ouyang and Zhang 2013, Yamagata et al. 2010, Yang et al. 2012, Xie et al. 2017). So far, at least ten hybrid sterility loci as gamete eliminator or pollen killer between $O$. sativa and $O$. glaberrima were identified. The cumulative effects of these sterility loci lead to complete male sterility in $\mathrm{F}_{1}$ hybrids. The one-locus allelic interaction model proposed by Kitamura (1962) can explain the genetic behavior of most hybrid sterility loci in indica-japonica and O. glaberrima$O$. sativa hybrids. According to this model and molecular evidence, Ikehashi and Araki (1986) proposed that the S5 locus, which is a major locus for hybrid sterility between indica and japonica subspecies, has at least three alleles, S5-n, S5-i and S5-j. In the heterozygotes of S5-iS5-j, female gametes with $S 5$-j are aborted due to the allelic interaction while the $S 5$-i gametes cannot be aborted, however such an abortion does not occur when $S 5-\mathrm{n}$ is present. The $S 5$-n 
allele is regarded as "neutral" sterility allele (wide compatibility gene). According to the neutral allele theory, the neutral allele S5-n was incorporated into indica or japonica varieties to overcome the sterility in intersubspecific hybrids (Ikehashi and Araki 1987). A number of widely compatible varieties in $O$. sativa were identified and confirmed to avoid the hybrid sterility at the corresponding locus ( $\mathrm{Lu}$ et al. 2004, Wan 1995).

Similarly, O. glaberrima and $O$. sativa varieties possess the genotype $S$-glab $S$-glab and $S$-sati $S$-sati, respectively, at a $S$ locus. Homozygotes of $S$-sati $S$-sati and $S$-glab $S$-glab show normal fertility, while the gametes of $S$-sati are aborted when the maternal plants have the genotype of $S$-sati $S$-glab in $O$. sativa background. However, there is no any report in regard to the neutral alleles in interspecific cross involving $O$. sativa and O. glaberrima. Heuer and Miezan (2003) crossed the wide compatibility varieties from $O$. sativa with $O$. glaberrima accessions in order to address the question whether the genes responsible for $F_{1}$ sterility in intersubspecific hybrids cause sterility in interspecific hybrids or not. The result showed that the "neutral" allele $S 5$-n improving fertility in intersubspecific hybrids had no fertility restoring effect in $\mathrm{F}_{1}$ interspecific hybrids.

It is thought that the reproductive isolation is the by-product of the accumulation for genetic differentiation between diverging populations (Dobzhansky 1970). Genetic differentiation provides the opportunities to increase the gene diversity during evolution. The accumulation of genetic differentiation can introduce the new function, divide the original function, or loss the original function (Lynch and Force 2000). For example, the loss-of-function allele of S27-niv ${ }^{s}$ which is composed of two inactive tandem mitochondrial ribosomal protein L27 genes (mtRPL27a and $m t R P L 27 b$ ), leads to $\mathrm{F}_{1}$ pollen sterility in the cross between $O$. sativa and O. nivara (Win et al. 2011). Moreover the null $S 27$-glum ${ }^{s}$, in which $m t R P L 27 a$ and $m t R P L 27 b$ are absent, also causes the $\mathrm{F}_{1}$ pollen sterility in the cross between $O$. sativa and O. glumaepatula (Yamagata et al. 2010). The evolution study of the nucleotide variation of the interspecific hybrid sterility $S 1$ gene showed that only a small genic changes occurred in the ancestral Oryza species and the diverged alleles have co-existed in the primitive gene pool of the genus Oryza (Guyot et al. 2011, Xie et al. 2017). It can be inferred that the action between different alleles produced by genetic differentiation can cause or eliminate reproductive isolation, which can be supported by the fact that the sterility of both pollen and spikelet as well as distorted segregation were restored when the OgTPR1 of $O$. glaberrima at $S 1$ was knocked out via CRISPR/Cas9 system (Xie et al. 2017).

The rice species with AA genome, which are classified into two cultivated species ( $O$. sativa and $O$. glaberrima), and six wild species $(O$. nivara, $O$. rufipogon, $O$. barthii, $O$. glumaepatula, $O$. longistaminata and $O$. meridionalis), are the most accessible genetic diversity resources, enabling us to look for neutral alleles among the different alleles at the known sterility loci. With the identification of some neutral alleles for certain hybrid sterility loci in intersubspecific hybrids, the phenomenon of the wide compatibility was also found in an Asian wild rice (O. rufipogon) strain (Hinata and Oka 1962). The neutral alleles at pollen sterility loci of cultivated rice from the ancestral $O$. rufipogon were also identified (Li et al. 2012, Liu et al. 2011, Shi et al. 2009).

Therefore, in this study, in order to detect whether the neutral allele which can restore the hybrid fertility in interspecific hybrid exists or not, an O. sativa subsp. japonica cultivar Dianjingyou 1 (DJY1) and its near isogenic lines (NILs) harboring the single sterility allele S1-glab, S19glab, S20-glab, S37-glab, S38-glab and S39-glab were crossed with $O$. sativa and its relatives with AA genome, respectively. Pollen fertility was analyzed in the paired $F_{1} S$ based on two seasons' result and genotypic segregation was also analyzed in some $F_{2}$ populations to confirm the results of pollen fertility investigation. The neutral alleles of $S 38-\mathrm{n}$ and S39-n were identified based upon the pollen fertility and genotypic segregation analysis for the first time.

\section{Materials and Methods}

\section{Genetic stocks}

Dianjingyou 1 (designated DJY1) and its six NILs denoted as NIL (S1), NIL (S19), NIL (S20), NIL (S37), NIL (S38) and NIL (S39) (Supplemental Table 1) carrying the single hybrid sterility allele $S 1$-glab, $S 19$-glab, $S 20$-glab, S37-glab, $S 38$-glab and S39-glab, respectively, from O. glaberrima were used as the test lines in this study. S1-glab and S37glab were identified as gamete eliminators on chromosome 6 and chromosome 1, respectively; the typical semi-sterility (about $50.0 \%$ ) for pollen and spikelet fertility was observed in $\mathrm{F}_{1}$ hybrids when the NILs (NIL (S1) and NIL (S37)) were crossed with their recurrent parent, DJY1 (Xu et al. 2014, Zhou et al. 2010). S19-glab, S20-glab, S38-glab and S39glab were identified as pollen killers on chromosome 3, 7, 4 and 12 , respectively; the pollen fertility of $F_{1}$ was semisterility while the spikelet fertility was normal when their NILs were crossed with their recurrent parent, DJY1 (Li et al. 2011, Xu et al. 2014).

Totally, 16 accessions, kindly provided by the International Rice Research Institute (IRRI) including two from $O$. glaberrima, one from O. rufipogon, three from O. nivara, one from $O$. glumaepatula, one from $O$. barthii, two from $O$. meridionalis and six from $O$. sativa accessions, were used as the tested lines (Table 1).

\section{Crossing and planting}

Six NILs and DJY1 (control line) were crossed as female parents (test lines) with the 16 tested lines in the Winter Crop Season of 2011 (November 2011-April 2012). Each tested line was crossed with both of DJY1 and its NILs to make pairs of test combinations. As the NILs and their recurrent parent DJY1 had the same background and the only difference was due to the specific chromosome fragment 
Table 1. Pollen fertility (\%) of the parents and $\mathrm{F}_{1} \mathrm{~s}$ between test lines and the tested lines in 2012

\begin{tabular}{|c|c|c|c|c|c|c|c|c|c|c|}
\hline Species & Subspecies/Ecotype & Accession & $\begin{array}{c}\text { DJY1 } \\
(97.8)^{a}\end{array}$ & $\begin{array}{c}\text { NIL }(S 1) \\
(98.0)\end{array}$ & $\begin{array}{c}\text { NIL }(S 19) \\
\quad(98.6)\end{array}$ & $\begin{array}{c}\text { NIL }(S 20) \\
(98.6) \\
\end{array}$ & $\begin{array}{c}\text { NIL }(S 37) \\
(98.3)\end{array}$ & $\begin{array}{c}\text { NIL }(S 38) \\
(98.1) \\
\end{array}$ & $\begin{array}{c}\text { NIL }(S 39) \\
(98.7)\end{array}$ & $\mathrm{LSD}_{0.05}$ \\
\hline O. sativa & Indica & Aswina (95.9) & 43.6 & 43.5 & 21.7 & 19.5 & 26.2 & 20.0 & 28.9 & 4.1 \\
\hline O. sativa & Temperate japinica & Tainung 67 (98.0) & 98.7 & 52.2 & 48.9 & 48.8 & 96.5 & 50.7 & 49.0 & 2.4 \\
\hline O. sativa & Tropical japonica & Azucena (96.5) & 21.0 & 21.1 & 17.7 & 7.9 & 22.1 & 14.4 & 34.9 & 2.6 \\
\hline O. sativa & Aromatic & Dom Sufid (98.0) & 25.1 & 2.2 & - & 14.8 & 4.8 & 30.5 & 20.7 & 3.3 \\
\hline O. sativa & Aus & Dular (90.8) & 94.3 & 43.5 & 47.8 & 25.2 & 41.6 & 48.8 & 37.5 & 1.5 \\
\hline O. sativa & Aus & Rayada (99.1) & 28.7 & 13.7 & 19.7 & 12.7 & 9.5 & 19.7 & 30.8 & 2.2 \\
\hline O. glaberrima & & IRGC102263 (94.7) & 0.2 & 0.3 & 0.0 & 0.4 & 0.9 & 0.0 & 0.0 & 1.0 \\
\hline O. glaberrima & & IRGC103469 (96.9) & 0.0 & 0.0 & 1.4 & 1.3 & 1.0 & 0.6 & 0.0 & 1.4 \\
\hline O. barthii & & Acc.104061 (92.3) & 6.3 & - & 2.7 & 3.1 & 1.6 & 2.9 & 2.6 & 4.0 \\
\hline O. glumaepatula & & Acc.103812 (93.2) & 74.2 & 39.3 & 25.2 & 20.8 & 44.1 & 41.8 & 40.7 & 5.0 \\
\hline O. meridionalis & & Acc.103322 (89.7) & 0.0 & 0.0 & 0.0 & 0.0 & 0.0 & 0.0 & 0.0 & - \\
\hline O. meridionalis & & Acc.104085 (87.2) & 0.0 & 0.0 & 0.0 & 0.0 & 0.0 & 0.0 & 0.0 & - \\
\hline O. nivara & & Acc.80453 (92.7) & 45.2 & 48.9 & 10.8 & 29.0 & 30.5 & 37.2 & - & 4.5 \\
\hline O. nivara & & Acc.80696 (94.1) & 40.8 & 23.8 & 23.1 & 7.4 & 21.8 & 22.9 & 22.7 & 2.2 \\
\hline O. nivara & & Acc.82012 (93.0) & 28.6 & 14.6 & 7.4 & 26.0 & 35.9 & 31.2 & 46.9 & 3.8 \\
\hline O. rifupogon & & PCR9607 (96.9) & 98.6 & 43.4 & 48.8 & 42.3 & 71.5 & 66.2 & 66.0 & 3.2 \\
\hline
\end{tabular}

${ }^{a}$ The pollen fertility of parents (\%).

harboring sterility $S$ allele, the effect of genetic background could be nearly ignored and the difference between DJY1 and the NILs was from the $S$ locus.

The $F_{1}$ hybrids were planted in the Late Crop Season of 2012 (May-September).

Some of the hybrid combinations were planted and investigated repeatedly in the Late Crop Season of 2015, and $\mathrm{F}_{1} \mathrm{~S}$ were self-pollinated to produce $\mathrm{F}_{2}$ populations.

All plant materials were planted at Winter Breeding Station, Yunnan Academy of Agricultural Sciences (YAAS), Sanya, Hainan Province, P. R. China.

\section{Phenotypic evaluation}

Pollen fertility for all parental lines, $F_{1}, F_{2}$ plants was investigated following the instructions of Zhu (1979). Pollen fertility was measured using anthers collected from spikelet at one to two days before anthesis and stored in 70\% ethanol (Doi et al. 1998). Three or four anthers per floret of each plant were mixed and stained with $1 \% \mathrm{I}_{2}-\mathrm{KI}$ solution, and more than 300 pollen grains were observed by light microscope. Sterile types were further classified as typical, spherical or stained abortion types (Li 1980). Three independent microscopic fields were scored for estimation of the percentage of the four types of pollen grains in each plant.

Spikelet fertility was scored as seed setting rate of two to five panicles on each plant.

Pollen and spikelet fertility data as percentage was transformed by function arcsin square root before the analysis but listed as percentage.

\section{Test for neutral allelic variation}

Identification method for the neutral alleles was described by Shi et al. (2009) with some modifications. It is supposed that DJY1, NIL and the tested line carrying the $S$-sati $S$-sati, $S$-glab $S$-glab and $S$-x $S$-x genotype, respectively, at a sterility $S$ locus. As we know that the $O$. glaberrima and $O$. sativa varieties possess genotype of $S$-glabS-glab and
$S$-sati $S$-sati, respectively; and in the case of heterozygotes $S$-satiS-glab, the gametes with $S$-sati is aborted due to a disharmonious allelic interaction while the $S$-glab gametes cannot be aborted, however such an abortion does not occur when $S$-n is present.

When the $\mathrm{F}_{1} \mathrm{~s}$ between a tested line $(S$-x) and NIL ( $S$-glab) showed the same pollen fertility level as the $\mathrm{F}_{1} \mathrm{~S}$ between the tested line $(S$-x) and DJY1 ( $S$-sati), it is suggested that the abortion is occurred neither between $S$-x and $S$-sati nor between $S$-x and $S$-glab at the target locus and the allele $(S$-x) carried by tested line could be regarded as a neutral allele $(S-n)$ at the locus $(S)$.

When the $\mathrm{F}_{1} \mathrm{~s}$ between a tested line $(S$-x $)$ and NIL $\left(S\right.$-glab) showed pollen fertility lower than the $\mathrm{F}_{1} \mathrm{~s}$ between the tested line $(S$-x) and DJY1 $(S$-sati), it means that the allele $(S$-x) carried in the tested line is compatible with $O$. sativa ( $S$-sati) and disharmonious interaction occurs between $S$-x and $S$-glab.

When the $\mathrm{F}_{1} \mathrm{~s}$ between a tested line $(S$-x $)$ and NIL ( $S$-glab) showed pollen fertility higher than that of $F_{1}$ s between the tested line $(S$-x) and DJY1 ( $S$-sati), it means that the allele $(S$-x) carried in the tested line is compatible with $O$. glaberrima $(S$-glab).

Molecular marker assay was also used to further confirm the existence of the neutral allele. The SSR (Simple Sequence Repeats) and Indel markers closely linked with sterility loci were selected to screen polymorphic markers between the pairs of test combinations. The polymorphic markers were used to genotype $F_{2}$ plants (Supplemental Table 2). The segregation ratio of closely linked markers to the respective locus would not deviate from the Mendelian ratio of 1:2:1 if abortion had not occurred. A chi-squared test was performed to determine whether the segregation ratio deviated from the Mendelian segregation ratio or not. If the detected allele $(S$-x) is a neutral allele $S$-n, the segregation in both of the $S$-x $\times S$-sati and $S$-x $\times S$-glab populations would fit the ratio of $1: 2: 1$. 


\section{Results}

The $F_{1}$ pollen fertility between the test lines and the tested lines

The pollen fertility of the $112 \mathrm{~F}_{1}$ cross combinations in 2012 were shown in Table 1.

Most of the hybrid combinations between the NILs and the tested lines gave lower pollen fertility than their controls. It is indicated that the sterility alleles from $O$. glaberrima cause the hybrid sterility not only between $O$. glaberrima and $O$. sativa but also between $O$. glaberrima and its wild relatives with AA genome.

The $F_{1}$ pollen fertility in the cross combinations of Aswina, Azucena, Dom Sufid and Rayada with DJY1 was low $(<50 \%)$ and the cause might be due to intersubspecific sterility within $O$. sativa.

The average pollen fertility obtained from the testcrosses between $O$. glaberrima, $O$. barthii, $O$. meridionalis and test lines was too low $(<10 \%)$ and there was no significant difference among them. It is indicated that more interspecific hybrid sterility loci were involved and a single sterillity allele from $O$. glaberrima had little contribution to the pollen fertility of interspecies hybrids.

\section{Identification of neutral alleles for the pollen sterility Test for allelic variation based on the pollen fertility in 2012}

$\mathrm{F}_{1}$ pollen fertility of most hybrids between tested lines and NILs was significantly lower than their controls, which suggests that most alleles $(S$-x) carried in the tested lines were compatible with $O$. sativa $(S$-sati) at the target $S$ loci (Table 1).

But, the average pollen fertility of $F_{1}$ obtained from the test crosses of Aswina, Azucena, Acc.80453 (O. nivara) with NIL (S1) was at the same level as the corresponding $\mathrm{F}_{1}$ between these tested lines and DJY1 (Table 1). It is suggested that the abortion was occurred neither between $S 1$-x and $S 1$-DJY1 nor between $S 1$-x and $S 1$-glab at the $S 1$ locus for these accessions. Thus, there may exist the neutral alleles at $S 1$ locus in the three tested lines, Aswina, Azucena and Acc.80453.

The $\mathrm{F}_{1}$ pollen fertility of all hybrids was significantly lower than the control in the testcrosses of the tested lines with NIL (S19) in 2012 (Table 1). It is indicated that the alleles of the tested lines at $S 19$ locus were compatible with $O$. sativa and no neutral allele among the tested lines for S19 locus were detected.

All the tested lines but Acc.82012 (O. nivara) gave lower $\mathrm{F}_{1}$ pollen fertility than their controls when crossed with NIL $(S 20)$. The pollen fertility $(26.0 \%)$ was at the same level as the control (28.6\%) when Acc.82012 was crossed with NIL $(S 20)$ in 2012 (Table 1). It can be inferred that Acc.82012 may carry a neutral allele at the $S 20$ locus.

The $\mathrm{F}_{1}$ pollen fertility from the crosses of NIL (S37) $\times$ Tainung 67 and NIL $($ S37) $\times$ Azucena were $96.5 \%$ and $22.1 \%$, respectively, which was not significantly different from their controls $(98.7 \%$ and $21.0 \%$, respectively). It is indicated that there may exist the neutral alleles at $S 37$ locus in Tainung 67 and Azucena. The pollen fertility (35.9\%) from the cross of NIL (S37) $\times$ Acc.82012 (O. nivara) was significantly higher than its control $(28.6 \%)$. It is suggested that there may exist a different allele in Acc.82012 (O. nivara) at $S 37$ locus compatible with $O$. glaberrima (Table 1).

For $S 38$ locus, as the $\mathrm{F}_{1}$ pollen fertility (30.5\%) from the cross of NIL $(S 38) \times$ Dom Sufid was significantly higher than its control (25.1\%), an O. glaberrima compatible allele in Dom Sufid was inferred. Since the $F_{1}$ pollen fertility $(31.2 \%)$ from the cross of NIL (S38) $\times$ Acc.82012 (O. nivara) was not significantly different from its control (28.6\%), a neutral allele in Acc.82012 was inferred for S38 locus, too (Table 1).

Two allelic variants compatible to $O$. glaberrima at $S 39$ locus were suggested as the $F_{1}$ pollen fertility from the crosses of Azucena, Acc.82012 (O. nivara) with NIL (S39) were significantly higher than the corresponding $\mathrm{F}_{1}$ between these tested lines and DJY1. Rayada was inferred to have a neutral allele at $S 39$ locus as the $\mathrm{F}_{1}$ pollen fertility from the crosses of NIL $($ S39) $\times$ Rayada was at the same level as the corresponding $\mathrm{F}_{1}$ in the cross of DJY1 $\times$ Rayada (Table 1). Test for allelic variation based on the pollen fertility in 2015

Based upon the result in 2012, part of the hybrids involved neutral alleles, $O$. glaberrima compatible alleles and data missing were planted and investigated again in 2015 .

The $\mathrm{F}_{1}$ pollen fertility in the cross of NIL $(S 1) \times$ Acc 80453 (O. nivara) was at the same level as the corresponding $\mathrm{F}_{1}$ in the cross of DJY1 $\times$ Acc. 80453 in 2012 and 2015, which suggests that Acc.80453 carried a neutral allele of S1-n. Similarly, Acc.82012 (O. nivara) may carry a neutral allele of S38-n and Rayada may carry an allele of S39-n (Table 2).

Acc.82012 and Azucena were deduced to carry the O. glaberrima compatible alleles at $S 37$ and $S 39$ loci, respectively, as the pollen fertility was significantly higher than their controls in 2012 (Table 1). However, the $F_{1}$ pollen fertility was at the same level as the control in 2015 (Table 2). It is suggested that the abortion was not occurred between $S$-x and $S$-glab at least, but more evidence is needed to identify whether the abortion occurred between $S$-x and $S$-DJY1 or not for these two accessions.

The $\mathrm{F}_{1}$ pollen fertility in the cross of NIL $(S 39) \times$ Acc.80453 (O. nivara) was missing in 2012, but at the same level as its control cross DJY1 $\times$ Acc. 80453 in 2015; more evidence is needed to support if there is a neutral allele at S39 locus in Acc.80453 (Table 2).

Confirmation of the neutral allelic variation using molecular marker assay

Because of the low seed setting rate and high shattering, only 3 pairs of test cross combination $\mathrm{F}_{2}$ populations were obtained. The polymorphic markers linked with $S 38$ in DJY1 $\times$ Acc.82012, NIL $($ S38) $\times$ Acc. 82012 showed a normal Mendelian segregation $(1: 2: 1)$ in their $F_{2}$ populations 
Table 2. Pollen fertility (\%) in the $\mathrm{F}_{1} \mathrm{~s}$ for some test crosses in 2015

\begin{tabular}{lccc}
\hline \hline Testcross & $\begin{array}{c}\text { Pollen fertility }(\%) \\
(\text { mean } \pm \text { SE) }\end{array}$ & $t$ value & P value \\
\hline DJY1 $\times$ Acc.80453 & $47.9 \pm 0.9$ & 1.868 & 0.185 \\
NIL $(S 1) \times$ Acc.80453 & $45.3 \pm 1.5$ & & \\
DJY1 $\times$ Acc.82012 & $29.7 \pm 1.1$ & 2.419 & 0.144 \\
NIL $($ S37) $\times$ Acc.82012 & $27.2 \pm 0.7$ & & \\
DJY1 $\times$ Acc.82012 & $29.7 \pm 1.1$ & 1.572 & 0.216 \\
NIL $($ S38 $) \times$ Acc.82012 & $24.4 \pm 3.1$ & & \\
DJY1 $\times$ Azucena & $20.5 \pm 3.0$ & 0.317 & 0.581 \\
NIL $($ S39 $) \times$ Azucena & $22.4 \pm 1.6$ & & \\
DJY1 $\times$ Rayada & $29.0 \pm 1.2$ & 0.384 & 0.539 \\
NIL $($ S39 $) \times$ Rayada & $29.9 \pm 1.0$ & & \\
DJY1 $\times$ Acc.80453 & $47.9 \pm 0.9$ & 2.846 & 0.114 \\
NIL $($ S39 $) \times$ Acc.80453 & $43.4 \pm 3.6$ & & \\
\hline
\end{tabular}

and the average pollen and spikelet fertility corresponding to the three genotypes showed non-significant difference (Table 3). Whereas the markers in the combination between DJY1 and NIL (S38) showed a significant deviation from the Mendelian segregation ratio $(1: 2: 1)$ and most of the individuals with semi-sterile pollen gains were heterozygous at the target markers and most of homozygotes had normal pollen fertility, indicating that there was a significant interaction between the alleles of DJY1 and NIL (S38) (Feng 2009, Xu et al. 2014). Therefore, it can be concluded that O. nivara Acc.82012 carries a neutral allele for pollen fertility at the $S 38$ locus.

In another two pairs of test combination, DJY1 $\times$ Rayada and NIL (S39) $\times$ Rayada, DJY1 $\times$ Acc.80453 (O. nivara) and NIL $($ S39) $\times$ Acc.80453, the polymorphic markers linked with $S 39$ showed normal Mendelian segregation $(1: 2: 1)$ in their $F_{2}$ populations and the average pollen and spikelet fertility corresponding to the three genotypes showed non-significant difference by the analysis of variance (Table 3), indicating that the alleles of Rayada and Acc.80453 interact with neither allele in DJY1 nor in NIL at $S 39$ locus. Whereas the markers in the correspondence combination of DJY1 1 NIL (S39) showed a significant

Table 3. Genotypic distribution of the linked molecular markers and their corresponding pollen and spikelet fertility in the $\mathrm{F}_{2}$ populations

\begin{tabular}{|c|c|c|c|c|c|c|c|c|c|c|c|}
\hline \multirow[t]{2}{*}{ Testcross } & \multirow[t]{2}{*}{ Marker } & \multirow[t]{2}{*}{ Genotypes } & \multirow{2}{*}{$\begin{array}{l}\text { Number } \\
\text { of plants }\end{array}$} & \multirow{2}{*}{$\begin{array}{c}\text { Pollen } \\
\text { fertility }(\%) \\
(\text { mean } \pm \text { SE) }\end{array}$} & \multirow{2}{*}{$\begin{array}{c}\text { Spikelet } \\
\text { fertility }(\%) \\
(\text { mean } \pm \text { SE) }\end{array}$} & \multicolumn{2}{|c|}{$\begin{array}{l}\text { Chi-squared test for } \\
\text { genotypes }\end{array}$} & \multicolumn{2}{|c|}{$\begin{array}{l}\text { Analysis of variance } \\
\text { for pollen fertility }\end{array}$} & \multicolumn{2}{|c|}{$\begin{array}{l}\text { Analysis of variance } \\
\text { for spikelet fertility }\end{array}$} \\
\hline & & & & & & $X^{2}$ value & $P$ value & F value & $P$ value & F value & $P$ value \\
\hline \multirow{6}{*}{$\begin{array}{l}\text { DJY1 } \times \\
\text { Rayada }\end{array}$} & RM8214 & $\mathrm{AA}^{a}$ & 39 & $63.6 \pm 6.3$ & $59.6 \pm 4.4$ & 0.399 & 0.819 & 0.716 & 0.493 & 0.440 & 0.646 \\
\hline & & $\mathrm{AB}$ & 69 & $58.4 \pm 6.8$ & $55.3 \pm 4.2$ & & & & & & \\
\hline & & $\mathrm{BB}$ & 35 & $71.5 \pm 9.1$ & $60.8 \pm 4.4$ & & & & & & \\
\hline & RM5568 & AA & 32 & $67.4 \pm 7.4$ & $54.3 \pm 4.9$ & 0.162 & 0.922 & 0.392 & 0.677 & 0.588 & 0.558 \\
\hline & & $\mathrm{AB}$ & 69 & $65.4 \pm 6.4$ & $59.4 \pm 3.8$ & & & & & & \\
\hline & & $\mathrm{BB}$ & 35 & $57.9 \pm 8.8$ & $61.8 \pm 4.9$ & & & & & & \\
\hline \multirow{6}{*}{$\begin{array}{l}\text { NIL }(S 39) \times \\
\text { Rayada }\end{array}$} & NJ5 & $\mathrm{AA}$ & 24 & $60.2 \pm 5.4$ & $55.0 \pm 5.9$ & 0.213 & 0.899 & 0.332 & 0.719 & 0.545 & 0.583 \\
\hline & & $\mathrm{AB}$ & 44 & $51.5 \pm 7.6$ & $56.5 \pm 4.3$ & & & & & & \\
\hline & & $\mathrm{BB}$ & 21 & $56.0 \pm 11.2$ & $48.8 \pm 6.9$ & & & & & & \\
\hline & RM5568 & $\mathrm{AA}$ & 23 & $60.9 \pm 5.1$ & $56.5 \pm 5.7$ & 0.121 & 0.941 & 0.764 & 0.472 & 1.035 & 0.362 \\
\hline & & $\mathrm{AB}$ & 44 & $48.6 \pm 7.5$ & $55.8 \pm 4.3$ & & & & & & \\
\hline & & $\mathrm{BB}$ & 24 & $50.6 \pm 9.5$ & $46.0 \pm 7.0$ & & & & & & \\
\hline \multirow{6}{*}{$\begin{array}{l}\text { DJY } 1 \times \\
\text { Acc. } 80453\end{array}$} & RM6371 & $\mathrm{AA}$ & 18 & $72.2 \pm 9.8$ & - & 1.700 & 0.427 & 1.082 & 0.349 & & \\
\hline & & $\mathrm{AB}$ & 25 & $64.8 \pm 8.5$ & - & & & & & & \\
\hline & & BB & 17 & $83.7 \pm 8.6$ & - & & & & & & \\
\hline & RM5568 & $\mathrm{AA}$ & 15 & $72.1 \pm 5.2$ & - & 1.000 & 0.607 & 0.267 & 0.979 & & \\
\hline & & $\mathrm{AB}$ & 25 & $62.9 \pm 8.8$ & - & & & & & & \\
\hline & & $\mathrm{BB}$ & 17 & $84.6 \pm 7.9$ & - & & & & & & \\
\hline \multirow{6}{*}{$\begin{array}{l}\text { NIL }(S 39) \times \\
\text { Acc. } 80453\end{array}$} & RM6371 & $\mathrm{AA}$ & 12 & $72.0 \pm 11.3$ & - & 0.679 & 0.712 & 0.169 & 0.846 & & \\
\hline & & $\mathrm{AB}$ & 31 & $66.7 \pm 6.8$ & - & & & & & & \\
\hline & & $\mathrm{BB}$ & 13 & $73.5 \pm 10.3$ & - & & & & & & \\
\hline & RM8214 & AA & 12 & $80.6 \pm 10.0$ & - & 0.125 & 0.939 & 0.518 & 0.602 & & \\
\hline & & $\mathrm{AB}$ & 25 & $65.1 \pm 8.0$ & - & & & & & & \\
\hline & & $\mathrm{BB}$ & 11 & $67.4 \pm 13.5$ & - & & & & & & \\
\hline \multirow{6}{*}{$\begin{array}{l}\text { DJY } 1 \times \\
\text { Acc. } 82012\end{array}$} & RM16260 & $\mathrm{AA}$ & 18 & $81.4 \pm 7.0$ & $53.1 \pm 8.0$ & 1.277 & 0.528 & 1.967 & 0.153 & 0.486 & 0.621 \\
\hline & & $\mathrm{AB}$ & 28 & $61.0 \pm 6.7$ & $57.4 \pm 6.1$ & & & & & & \\
\hline & & $\mathrm{BB}$ & 19 & $73.2 \pm 6.5$ & $49.1 \pm 5.6$ & & & & & & \\
\hline & W-9 & AA & 22 & $83.6 \pm 5.7$ & $52.1 \pm 6.2$ & 1.886 & 0.390 & 2.378 & 0.105 & 1.417 & 0.256 \\
\hline & & $\mathrm{AB}$ & 30 & $61.8 \pm 6.3$ & $59.8 \pm 4.3$ & & & & & & \\
\hline & & $\mathrm{BB}$ & 18 & $70.5 \pm 6.7$ & $48.3 \pm 5.6$ & & & & & & \\
\hline $\operatorname{NIL}(S 38) \times$ & RM16335 & $\mathrm{AA}$ & 14 & $78.7 \pm 17.0$ & $37.5 \pm 23.8$ & 1.636 & 0.441 & 0.613 & 0.556 & 0.118 & 0.889 \\
\hline \multirow[t]{5}{*}{ Acc. 82012} & & $\mathrm{AB}$ & 22 & $53.8 \pm 16.5$ & $43.9 \pm 5.2$ & & & & & & \\
\hline & & $\mathrm{BB}$ & 8 & $56.1 \pm 13.8$ & $45.2 \pm 8.7$ & & & & & & \\
\hline & W-9 & $\mathrm{AA}$ & 16 & $56.1 \pm 13.8$ & $45.6 \pm 8.7$ & 2.640 & 0.267 & 0.017 & 0.983 & 0.834 & 0.445 \\
\hline & & $\mathrm{AB}$ & 26 & $58.2 \pm 13.2$ & $38.7 \pm 5.1$ & & & & & & \\
\hline & & $\mathrm{BB}$ & 8 & $60.3 \pm 19.9$ & $54.2 \pm 11.7$ & & & & & & \\
\hline
\end{tabular}

\footnotetext{
${ }^{a}$ AA symbols the genotype of homozygote of test lines, $\mathrm{AB}$ symbols the genotype of heterozygote and BB symbols the genotype of homozygote of tested lines.
} 
deviation from the Mendelian segregation ratio (1:2:1) and most of the individuals with semi-sterile pollen gains were heterozygous at the target markers and most of homozygotes had normal pollen fertility, indicating that there was a significant interaction between the alleles of DJY1 and NIL (S39) (Feng 2009, Xu et al. 2014). It can be inferred that Rayada and Acc.80453 (O. nivara) carry neutral allele $S 39$-n at the $S 39$ locus.

\section{The different alleles existed at the hybrid sterility loci}

The pollen fertility of $\mathrm{F}_{1}$ plants at the specific sterility $S$ locus was varied among the different tested lines (Table 1). In order to estimate the allelic variation by the pollen fertility, the variation degree $(d)$ was calculated based on an algebraic formula as below (Zhang et al. 1993).

$$
d=\left(f_{2}-f_{1}\right) / f_{1}
$$

$f_{1}$ and $f_{2}$ represent the pollen fertility percentage of $F_{1} \mathrm{~s}$ when one tested line crossed with DJY1 and a NIL, respectively. The allelic variation degree was estimated based on the pollen fertility of the $\mathrm{F}_{1}$ plants of the cross combinations including $O$. sativa, O. glumaepatula, $O$. nivara and $O$. rifupogon (Table 4). The negative value means that the allele was compatible with $O$. sativa $(S$-sati) and the positive value means that the allele was compatible with O. glaberrima ( $S$-glab).

The distribution of allelic variation degree was different at different sterility loci and ranged from -0.914 to 0.6608 with different lines tested. Three allelic variation types at $S 1$ locus were observed. Two $O$. sativa accessions, Aswina and Azucena, and one $O$. nivara accession, Acc.80453, had the similar allele variation degree near 0.000 at $S 1$ locus. Eight tested lines had the similar allele variation degree between -0.4000 and -0.6000 , while Dom Sufid had another variation degree with much different value of -0.9140 (Table 4, Fig. 1). It is suggested that there are at least two allelic

Table 4. The allelic variation degree at the six hybrid sterility loci in 11 tested lines

\begin{tabular}{|c|c|c|c|c|c|c|c|c|}
\hline Species & Subspecies/Ecotype & Accession & NIL (S1) & NIL (S19) & NIL $(S 20)$ & NIL (S37) & NIL $(S 38)$ & NIL (S39) \\
\hline O. sativa & Indica & Aswina & -0.0023 & -0.5017 & -0.5524 & -0.4000 & -0.5425 & -0.3376 \\
\hline O. sativa & Temperate japinica & Tainung 67 & -0.4712 & -0.5048 & -0.5052 & -0.0222 & -0.4857 & -0.5032 \\
\hline O. sativa & Tropical japonica & Azucena & 0.0029 & -0.1563 & -0.6260 & 0.0519 & -0.3144 & 0.6608 \\
\hline O. sativa & Aromatic & Dom Sufid & -0.9140 & - & -0.4090 & -0.8096 & 0.2162 & -0.1744 \\
\hline O. sativa & Aus & Dular & -0.5385 & -0.4928 & -0.7329 & -0.5623 & -0.4828 & -0.6021 \\
\hline O. sativa & Aus & Rayada & -0.5215 & -0.3146 & -0.5588 & -0.6704 & -0.3132 & 0.0750 \\
\hline O. glumaepatula & & Acc. 103812 & -0.4710 & -0.6608 & -0.7203 & -0.4054 & -0.4376 & -0.4518 \\
\hline O. nivara & & Acc. 80453 & 0.0811 & -0.7619 & -0.3595 & -0.3261 & -0.1786 & - \\
\hline O. nivara & & Acc. 80696 & -0.4168 & -0.4335 & -0.8199 & -0.4663 & -0.4389 & -0.4430 \\
\hline O. nivara & & Acc. 82012 & -0.4893 & -0.7405 & -0.0915 & 0.2522 & 0.0901 & 0.6395 \\
\hline O. rifupogon & & PCR9607 & -0.5596 & -0.5050 & -0.5711 & -0.2751 & -0.3289 & -0.3310 \\
\hline LSD0.05 & & & 0.1162 & & & & & \\
\hline
\end{tabular}

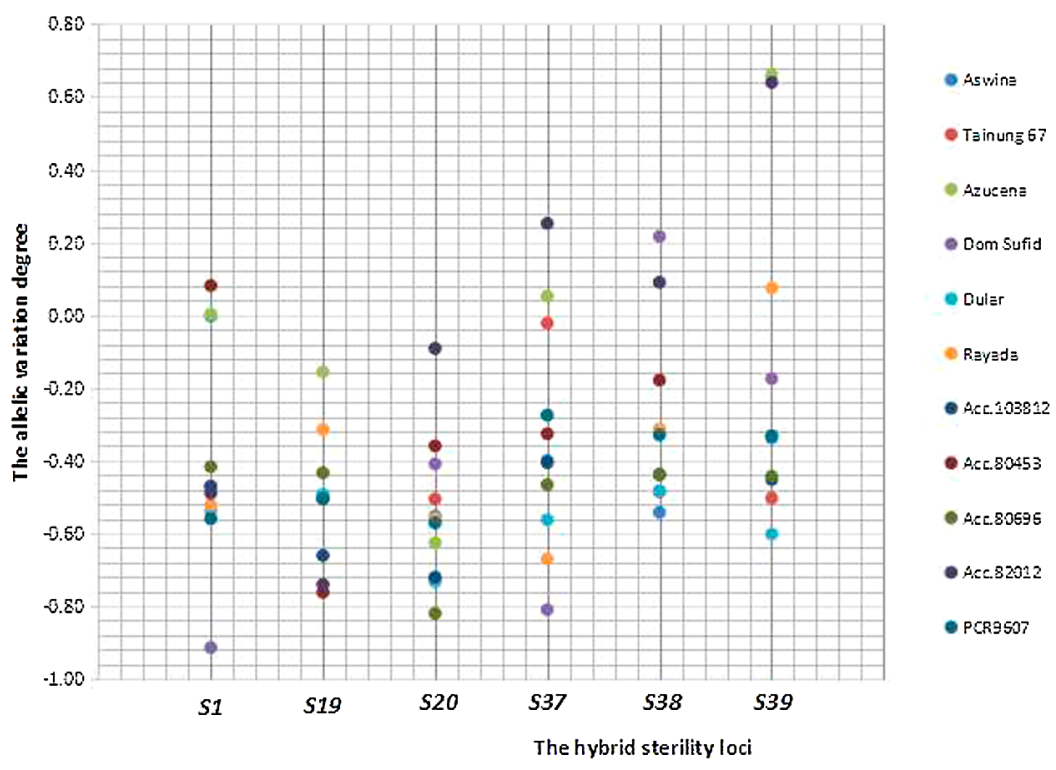

Fig. 1. The allelic variation degree at six hybrid sterility loci among 11 tested lines. The horizontal axis was divided into six loci and each locus represents a different sterility locus from Oryza glaberrima. The allelic variation degree of 11 tested lines were divided into three groups at $S 1$ locus, disperse from -0.1563 to -0.7619 at $S 19$ locus, two groups at $S 20$ locus; but more than three groups were observed at $S 37(\mathrm{t}), S 38(\mathrm{t})$ and S39(t) loci. 
variants compatible with $O$. sativa at $S 1$ locus.

The complex variation was also observed at other sterility loci. The allelic variations of all the tested lines were compatible with $O$. sativa at $S 19$ locus with the range from -0.1563 to -0.7619 . The sets of $O$. sativa compatible variations ranged from -0.3595 to -0.8199 were detected at $S 20$ locus in 11 tested lines but one neutral allelic variation from O. nivara accession Acc.82012 with the variation degree of -0.0915 . The neutral and $O$. glaberrima compatible allelic variations were also detected at $S 37, S 38, S 39$ loci in addition to a set of $O$. sativa compatible allelic variations. The biggest variation was ranged from -0.6021 to 0.6608 at $S 39$ locus including the largest $O$. glaberrima compatible allelic variation degree (Table 4, Fig. 1).

The result implied that the allelic variation is much more complex than three alleles model of neutral, O. sativa compatible and $O$. glaberrima compatible at a target $S$ locus. Of course, the allelic variation analyzed based on the pollen fertility of 2012 needs further confirmation, and the further study is ongoing.

\section{Discussion}

Near-isogenic lines can be used for detecting the neutral allelic variation

Testcross is the major method for identifying neutral allele. However, the multiple sterility loci between $O$. sativa and $O$. glaberrima influence the accuracy of the test results, as the result it is nearly impossible to detect any single neutral allele between these two species. From this viewpoint, DJY1 and its NILs carrying the single sterility allele from O. glaberrima, which had a similar genetic background, were used as the test lines in our study. If a tested line is crossed with DJY1 and one of its NILs simultaneously, the difference for the pollen fertility in the pair of testcross was caused by allelic interaction at the target $S$ locus since the difference is from the allelic difference between DJY1 and a NIL. Thus, the effects of $S$-x carried in the tested line could be accurately determined through this experimental design used in the study.

In this study, we tried to cross DJY1 and its NILs with AA genome species accessions for detecting the neutral alleles. All the $\mathrm{F}_{1}$ seeds of crosses were got but $O$. longistaminata as the embryo abortion occurred early between $O$. sativa and $O$. longistaminata. The results showed that the neutral alleles of $S 38$-n and S39-n responding to the interspecific hybrid sterility between $O$. sativa and $O$. glaberrima were identified in Asian cultivated rice and $O$. nivara accessions for the first time. Our preliminary study also detected the neutral alleles for $S 1$ and $S 37$ loci based upon the pollen fertility from $O$. nivara accessions Acc.80453 and Acc.82012, which needs more molecular evidence to support. Considering $S 1$ and $S 37$ loci were gamete eliminators (Li et al. 2011, $\mathrm{Xu}$ et al. 2014), it is difficult to get $\mathrm{F}_{2}$ population if other hybrid sterility locus controlling the embryo sac is also involved. Developing the near isogenic lines carried the alleles of tested lines is necessary to further clear the allelic variation and understand the genetic action of allelic interaction.

The allele compatible with $O$. glaberrima was not identified at the molecular level in this study. For the allele compatible with $O$. glaberrima, the segregation ratio of closely linked markers to the sterility locus would deviate from the Mendelian ratio in the $\mathrm{F}_{2}$ population between tested line and DJY1 but fit the Mendelian ratio in the $F_{2}$ population between tested lines and near-isogenic lines, as the disharmonious interaction occurred not between $S$-x and $S$-glab but between $S$-x and $S$-sati.

The study for the allelic variation of sterility gene may open the ways to overcome the interspecific hybrid sterility

Since the sterility of interspecific hybrid is caused by allelic interaction at a small number of loci, it is possible to overcome it by introgressing the major sterility alleles to Asian cultivated rice as bridge parent (Tao et al. 2003). Based upon the known genetic information, if sterility alleles in a given $O$. sativa background were substituted by the neutral alleles or the corresponding alleles from O. glaberrima, it would be possible to overcome the interspecific hybrid sterility between $O$. sativa and $O$. glaberrima (Heuer and Miézan 2003, Tao et al. 2003). Compatibility alleles $S 5$-n, $S a$-n, $S b$-n, $S d$-n, $S e$-n in intersubspecific hybrid have been identified (Ikehashi and Araki 1986, Li et al. 2012, Long et al. 2008). The utility of these neutral alleles will have a strong effect on overcoming the indica-japonica hybrid sterility along with the origin and evolution study of rice.

The utilization of the compatible alleles with O. glaberrima from $O$. sativa and other wild relatives of cultivated rice can be another way to overcome the interspecific hybrid sterility between $O$. sativa and $O$. glaberrima. These accessions with neutral or compatible allele with $O$. glaberrima could be important germplasm resources for overcoming the interspecific hybrid sterility. Considering the interspecific hybrid sterility is the result of the allelic interactions from multiple sterility loci, the next step of research is to pyramid the neutral or compatible alleles in the Asian cultivated rice as the bridge parents, but it is necessary to understand the nature of interaction between them in advance.

The allelic variation of sterility gene may enable us to understand the origin and evolution of Genus Oryza

The relationship between reproductive isolation and the genetic divergence is one of the interest areas of evolutionary biology. The hybrid sterility gene is one of the speciation genes involved in the formation of new species or subspecies. Some of the sterility loci from different hybrid combinations had the same chromosome location and same genetic action. For example, $S 1$ was reported in many interspecific hybrid crosses between $O$. sativa and its AA genome relatives including $O$. glaberrima, $O$. longistaminata, $O$. rufipogon, O. nivara and $O$. barthii (Chen et al. 2009, Sano et al. 1979, Yang et al. 2016). The co-linear relationship was also observed between $S 20$ from $O$. glaberrima 
and S56(t) from O. glumaepatula (Doi et al. 1999, Zhang et al. 2018), between $S 21$ from $O$. glaberrima and $O$. rufipogon, and $S 23$ from O. glumaepatula on chromosome 7 (Doi et al. 1999, Miyazaki et al. 2007, Sobrizal et al. 2000a), between $S 22$ from O. glumaepatula and $S 29(\mathrm{t})$ from O. glaberrima on chromosome 2 (Hu et al. 2006, Sobrizal et al. 2000b). The intersubspecific sterility locus S25 shared the same chromosome location with $S 39(\mathrm{t})$ from $O$. glaberrima and $S 36$ from $O$. nivara on the end of short arm of chromosome 12 based on the comparison of map positions (Win et al. 2009, Xu et al. 2014). These sterility loci with good co-linear might be orthologous loci with sets of alleles. The allelic variation at the hybrid sterility loci provides the clue for the origin and evolution of cultivated rice. In our study, the great range of pollen fertility of $F_{1}$ plants at the specific sterility $S$ locus was observed among different tested lines. It is implied that there are not only three alleles of neutral, $O$. sativa and $O$. glaberrima at a target $S$ locus but also a set of different alleles in the tested lines. The evolutionary study of such special group of genes enables us understanding the origin and variation events between incipient species. The molecular evolution of $S 5$ suggested that the $S 5-n$ sequence was not biased and $S 5-n$ evolved simultaneously by parallel evolution in wild relatives and cultivated rice (Tong et al. 2011), and the independent origins of indica and japonica subspecies was deduced (Du et al. 2011). The antiquity tri-allelic system, such as $S 5$ comprised three types of alleles of $S 5-i, S 5-j, S 5-n$, acts as an important factor in the genetic differentiation and provides an excellent model system for studying the evolutionary processes of reproductive isolation and speciation (Du et al. 2011). Further study of these neutral genes and depth excavation of germplasm resources will be conducive to enrich interspecific hybridization breeding strategies and enable us to order species pairs by their time of divergence.

The $\mathrm{F}_{1}$ pollen fertility was low $(<10 \%)$ in some crosses, including $O$. sativa $\times O$. glaberrima, $O$. sativa $\times O$. barthii, $O$. sativa $\times O$. meridionalis, indicating that more interspecific hybrid sterility loci were involved. One locus allelic variation between test lines and tested lines cannot be detected from several loci affects and interaction. One of the ways to detect the allelic variation at sterility loci for these materials is to raise near-isogenic lines based upon the cloning and sequence analysis of sterility loci, to understand the relationship between allelic variation and interspecific hybrid sterility so as to understand the relationship between speciation and hybrid sterility.

\section{Acknowledgment}

This research was funded by grants from the National Natural Science Foundation of China (Grant Nos. 31000704, U1036605, U1502265).

\section{Literature Cited}

Chang, T.T. (1976) The origin, evolution, cultivation, dissemination, and diversification of Asian and African rices. Euphytica 25: 425441.

Chen, J.J., J.H.Ding, Y.D. Ouyang, H.Y.Du, J.Y.Yang, K.Cheng, J.Zhao, S.Q.Qiu, X.L.Zhang, J.L. Yao et al. (2008) A triallelic system of $S 5$ is a major regulator of the reproductive barrier and compatibility of indica-japonica hybrids in rice. Proc. Natl. Acad. Sci. USA 105: 11436-11441.

Chen, Z.W., F.Y.Hu, P. Xu, J. Li, X.N. Deng, J.W.Zhou, F. Li, S.N. Chen and D.Y.Tao (2009) QTL analysis for hybrid sterility and plant height in interspecific populations derived from a wild rice relative, Oryza longistaminata. Breed. Sci. 59: 441-445.

Dobzhansky, T. (1970) Genetics of the evolutionary process. Columbia Univ. Press, NY, London.

Doi, K., A. Yoshimura and N. Iwata (1998) RFLP mapping and QTL analysis of heading date and pollen sterility using backcross population between Oryza sativa L. and Oryza glaberrima Steud. Breed. Sci. 48: 395-399.

Doi, K., K. Taguchi and A. Yoshimura (1999) RFLP mapping of S20 and $S 21$ for $\mathrm{F}_{1}$ pollen semi-sterility found in backcross progeny of Oryza sativa and O. glaberrima. Rice Genet. Newsl. 16: 65-68.

Du,H.Y., Y.D. Ouyang, C.J.Zhang and Q.F.Zhang (2011) Complex evolution of $S 5$, a major reproductive barrier regulator, in the cultivated rice Oryza sativa and its wild relatives. New Phytol. 191: 275-287.

Feng, S.F. (2009) Study on uncovering and mapping of inter-specific hybrid sterility genes between Oryza sativa and O. glaberrima. Master Degree thesis, Yunnan Agricultural University, Kunming, P.R.China.

Guyot, R., A. Garavito, F. Gavory, S. Samain, J. Tohme, A. Ghesquière and M. Lorieux (2011) Patterns of sequence divergence and evolution of the $S 1$ orthologous regions between Asian and African cultivated rice species. PLoS ONE 6: 260-260.

Heuer, S. and K.M. Miézan (2003) Assessing hybrid sterility in Oryza glaberrima $\times O$. sativa hybrid progenies by PCR marker analysis and crossing with wide compatibility varieties. Theor. Appl. Genet. 107: 902-909.

Hinata, K. and H.I. Oka (1962) A survey of hybrid sterility relationship in the Asian forms of Oryza perennis and O. sativa. Jpn. J. Genet. 37: $314-328$.

Hu, F., P. Xu, X. Deng, J.Zhou, J.Li and D. Tao (2006) Molecular mapping of a pollen killer gene $S 29(\mathrm{t})$ in Oryza glaberrima and colinear analysis with $S 22$ in O. glumaepatula. Euphytica 151: 273278.

Ikehashi, H. and H.Araki (1986) Genetics of $F_{1}$ sterility in remote crosses of rice. In: International Rice Research Institute-IRRI (ed.) Rice Genetics, IRRI, Manila, pp. 119-130.

Ikehashi, H. and H. Araki (1987) Screening and genetic analysis of wide compatibility in $\mathrm{F}_{1}$ hybrid of distant crossed in rice (Oryza sativa L.). In: Asai, T. (ed.) Technical Bulletin of the Tropical Agriculture Research Center, Tropical Agriculture Research Center, Tsukuba, pp. 1-79.

Kitamura, E. (1962) Genetic studies on sterility observed in hybrids between distantly related varieties of rice, Oryza sativa L. Bulletin of the Chugoku National Agricultural Experiment Station 8: 141205.

Kubo, T., T. Takashi, M.Ashikari, A. Yoshimura and N. Kurata (2015) Two tightly linked genes at the hsal locus cause both $\mathrm{F}_{1}$ and $\mathrm{F}_{2}$ hybrid sterility in rice. Mol. Plant 9: 221-232. 
Li, J., P. Xu, J. Zhou, F. Hu, X. Deng, Z. Chen and D. Tao (2011) Molecular mapping of sterility QTLs $q S S-3, q S S-6 a$ and $q S S-7$ as single Mendelian factors via NIL strategy. Rice Sci. 18: 110-115.

Li,J.Q., M.Q. Shahid, J.H.Feng, X.D.Liu, X.J.Zhao and Y.G.Lu (2012) Identification of neutral alleles at pollen sterility gene loci of cultivated rice (Oryza sativa L.) from wild rice (O. rufipogon Griff.). Plant Syst. Evol. 298: 33-42.

Li,Z.B. (1980) A preliminary discussion about the classification of male sterile lines of rice in China. Acta Agron. Sin. 6: 17-26.

Liu, B., J.Q.Li, X.D.Liu, M.Q. Shahid, L.G. Shi and Y.G.Lu (2011) Identification of neutral genes at pollen sterility loci $S d$ and $S e$ of cultivated rice (Oryza sativa) with wild rice (O. rufipogon) origin. Genet. Mol. Res. 10: 3435-3445.

Long, Y.M., L.F.Zhao, B.X.Niu, J.Su, H.Wu, Y.L.Chen, Q.Y.Zhang, J.X. Guo, C.X.Zhuang, M.T. Mei et al. (2008) Hybrid male sterility in rice controlled by interaction between divergent alleles of two adjacent genes. Proc. Natl. Acad. Sci. USA 105: 18871-18876.

Lu, C.G., J.S.Zou and H.Ikehashi (2004) Developing rice lines possessing neutral alleles at sterility loci to improve the width of compatibility. Plant Breed. 123: 98-100.

Lynch, M. and A.G. Force (2000) The origin of interspecific genomic incompatibility via gene duplication. Am. Nat. 156: 590-605.

Miyazaki,Y., K. Doi, H. Yasui and A. Yoshimura (2007) Identification of a new allele of $F_{1}$ pollen sterility gene, $S 21$, detected from the hybrid between Oryza sativa and O. rufipogon. Rice Genet. Newsl. 23: 36-38.

Mizuta, Y., Y.Harushima and N. Kurata (2010) Rice pollen hybrid incompatibility caused by reciprocal gene loss of duplicated genes. Proc. Natl. Acad. Sci. USA 107: 20417-20422.

Ohmido, N. and K. Fukui (1995) Cytolofical studies of African cultivated rice, Oryza glaberrima. Theor. Appl. Genet. 91: 212-217.

Ouyang, Y.D. and Q.Zhang (2013) Understanding reproductive isolation based on the rice model. Annu. Rev. Plant Biol. 64: 111-135.

Sano, Y., Y.E. Chu and H.I. Oka (1979) Genetic studies of speciation in cultivated rice, 1. Genic analysis for the $F_{1}$ sterility between O. sativa L. and O. glaberrima Steud. Jpn. J. Genet. 54: 121-132.

Sano, Y. (1990) The genic nature of gamete eliminator in rice. Genetics 125: 183-191.

Shi,L.G., X.D.Liu, B.Liu, X.J.Zhao, L.Wang, J.Q.Li and Y.G.Lu (2009) Identifying neutral allele $S b$ at pollen-sterility loci in cultivated rice with Oryza rufipogon origin. Chin. Sci. Bull. 54: 3813 3821.

Sobrizal, Y.Matsuzaki, P.L.Sanchez, K.Ikeda and A. Yoshimura (2000a) Identification of a gene for male gamete abortion in backcross progeny of Oryza sativa and Oryza glumaepatula. Rice Genet. Newsl. 17: 59-61.

Sobrizal, Y.Matsuzaki, P.L.Sanchez, K.Ikeda and A. Yoshimura (2000b) Mapping of $F_{1}$ pollen semi-sterility gene found in backcross progeny of Oryza sativa L. and Oryza glumaepatula Steud. Rice Genet. Newsl. 17: 61-63.

Tanksley, S.D. and S.R.McCouch (1997) Seed banks and molecular maps: unlocking genetic potential from the wild. Science 277:
1063-1066.

Tao, D., P. Xu, J. Li, Y. Yang, J.Zhou, F. Hu and M.P. Jones (2003) Studies on hybrid sterility inheritance and mapping of sterile genes among near-isogenic lines derived from interspecific hybrid between cultivated rice species Oryza sativa L. and O. glaberrima Steud. Chinese J. Rice Sci. 17: 11-15.

Tong, J.F., Y.H.Li, Y.X. Yang, M.Q.Shahid, Z.X.Chen, L.Wang, J.Q.Li, X.D.Liu and Y.G.Lu (2011) Molecular evolution of rice $S 5^{n}$ and functional comparison among different sequences. Chin. Sci. Bull. 56: 2016-2024.

Wan, J.M. (1995) Analysis of hybrid sterility gene loci for hybrid rice breeding and understanding of varietal differentiation. $\mathrm{PhD}$ thesis, Kyoto University, Kyoto.

Win, K.T., T. Kubo, Y. Miyazaki, K. Doi, Y. Yamagata and A. Yoshimura (2009) Identification of two loci causing $F_{1}$ pollen sterility in interand intraspecific crosses of rice. Breed. Sci. 59: 411-418.

Win, K.T., Y.Yamagata, Y. Miyazaki, K. Doi, H. Yasui and A. Yoshimura (2011) Independent evolution of a new allele of $F_{1}$ pollen sterility gene $S 27$ encoding mitochondrial ribosomal protein L27 in Oryza nivara. Theor. Appl. Genet. 122: 385-394.

Xie, Y., P.Xu, J.Huang, S.Ma, X.Xie, D.Tao, L.Chen and Y.G.Liu (2017) Interspecific hybrid sterility in rice is mediated by $O g T P R 1$ at the $S 1$ locus encoding a peptidase-like protein. Mol. Plant 10: $1137-1140$.

Xu,P., J.Zhou, J.Li, F.Hu, X.Deng, S.Feng, G.Ren, Z.Zhang, W. Deng and D. Tao (2014) Mapping three new interspecific hybrid sterile loci between Oryza sativa and O. glaberrima. Breed. Sci. 63: 476-482.

Yamagata, Y., E. Yamamoto, K.Aya, K.T.Win, K. Doi, Sobrizal, T. Ito, H.Kanamori, J.Z.Wu, T. Matsumoto et al. (2010) Mitochondrial gene in the nuclear genome induces reproductive barrier in rice. Proc. Natl. Acad. Sci. USA 107: 1494-1499.

Yang, J., X.Zhao, K.Cheng, H.Du, Y. Ouyang, J.Chen, S.Qiu, J.Huang, Y. Jiang, L. Jiang et al. (2012) A killer-protector system regulates both hybrid sterility and segregation distortion in rice. Science 337: 1336-1340.

Yang, Y., J.W.Zhou, J.Li, P.Xu, Y.Zhang and D.Y.Tao (2016) Mapping QTLs for hybrid sterility in three AA genome wild species of Oryza. Breed. Sci. 66: 367-371.

Zhang, G.Q., Y.G. Lu, G.F. Liu, J.C. Yang and H.Zhang (1993) Genetic studies of the hybrid sterility in cultivated rice (Oryza sativa). Acta Genet. Sin. 20: 541-551.

Zhang, Y., J.Zhou, J.Li, Y. Yang, P.Xu and D. Tao (2018) Mapping of S56(t) responsible for interspecific hybrid sterility between Oryza sativa and Oryza glumaepatula. Breed. Sci. 68: 242-247.

Zhou, J., P.Xu, X.Deng, J.Li, F.Hu, G.Ren, Z.Zhang, Y.Luan, W. Deng, Z. Zhao et al. (2010) Genetic dissection of a chromosomal region conferring hybrid sterility using multi-donors from Oryza glaberrima. Euphytica 175: 395-407.

Zhu, Y.G. (1979) A preliminary discussion about the classification of male sterile lines of rice in China. Acta Agron. Sin. 6: 17-26. 